\title{
El servicio de consultoría estratégica en la gestión de sistemas de información
}

\author{
Zunilka Limonta Favier \\ Evelyn Milagro Otero Caballero \\ Daymara Mediaceja Corona \\ Centro de Información y Gestión Tecnológica - Cuba \\ Briseys Calvo Coello \\ Universidad de Guantánamo - Cuba
}

\section{ANÁLISIS}

\begin{abstract}
Resumen
Se presentan las características de un servicio que posibilita el diseño, gestión, innovación e implementación de sistemas de información en organizaciones académicas, de bienes y servicios etc. que se tiene concebido como asesoría a entidades que lo requieran atendiendo a sus necesidades ya sean externas o internas. Se brindan algunas recomendaciones para mejorar el trabajo en sistemas de información y su gestión. Este trabajo demuestra cómo se puede brindar un servicio diferenciado según las características de cada institución.
\end{abstract}

Palabras clave

Sistemas de información ; Servicios de información ; Consultorías ; Proyectos

\section{The service of strategic consultancy in information systems management}

\begin{abstract}
We present a service that enables the design, management, innovation and implementation of information systems in academic, service, economic etc. which is intended as advice to entities that require attending to their needs whether external or internal. They offer some recommendations to improve the work in information systems and management. This paper shows how to provide differentiated services according to the characteristics of each institution.
\end{abstract}

Keywords

Information systems ; Information services ; Consulting ; Proyects

\section{Introducción}

Mediante el uso de Nuevas Tecnologías de la Información y el Conocimiento (NTICs) se garantiza el acceso a información, que puede ser la base de los nuevos descubrimientos e innovaciones necesarios para la supervivencia del hombre; por eso es tan habitual en estos días, escuchar hablar en materia de información, de cambio de época en lugar de época de cambios.

La información se encuentra disponible en diferentes fuentes como son: la literatura de ciencia y técnica, las patentes, los libros, los expertos, Internet y se elabora información para compartir el conocimiento, alfabetizarse informacionalmente, para apoyar las decisiones de los directivos, minimizar los riesgos y predecir el futuro 
empresarial. Actualmente las organizaciones con o sin acceso a las ventajas que proporcionan las NTICs realizan diferentes procesos con el fin de cumplir sus objetivos organizacionales, para posicionarse en el mercado y superar a su competencia, algunos de ellos son: gestión del conocimiento, vigilancia tecnológica, inteligencia empresarial y la gestión de sistemas de información. Además las entidades, instituciones u organismos cuentan con diversos productos/servicios que se perfeccionan e incrementan con el objetivo de ponerlos a disposición de sus usuarios y/o clientes y así satisfacer sus necesidades.

Los CIGET no está exento de esta realidad, en ellos se diseñan y prestan productos/servicios y se realizan diversos procesos, de manera tal que gestionan su propio sistema y asesoran a otras entidades para ayudarlos a gestionar los suyos. Por investigaciones previas realizadas en la comunidad empresarial, como parte de un estudio de mercado y en atención a las necesidades de los clientes, se diseñó un servicio que respondiera a las deficiencias, insuficiencias o inexistencia de un sistema que trate la información de forma adecuada, que respete los canales o flujos diseñados para esta. Fue ideado para su aplicación en tres etapas, en las cuales se analizan las características del cliente, se hace un levantamiento de personal, actividades, equipamiento, local, materiales etc., con que cuenta la entidad, institución u organismo.

Posteriormente se diseña un servicio que cumpla con los requisitos del correcto flujo organizacional, por tal razón siempre será necesario la elaboración del diagrama de flujo informacional. Preveer un producto que responda a las necesidades, siendo este: una intranet corporativa, un sitio Web, una multimedia, un sistema de archivo, un centro documental etc. Por último se realiza una prueba piloto, correspondiente a la puesta en marcha del servicio, velar por su correcto funcionamiento y limar errores o deficiencias en caso que sean encontradas.

En cualquier institución, empresa u organismo, debe existir un sistema de información que reúna organizadamente los documentos que se circulan en ellas. Esta información es tratada por todos los trabajadores, pero solo uno se encarga de mantener al día, la disponibilidad y orden de la misma.

\section{Conceptualización}

El diccionario de la lengua española en su vigésima segunda edición define:

Gestión: Acción y efecto de gestionar. Acción y efecto de administrar. Dentro de los negocios se reconoce como un cuasicontrato que se origina por el cuidado de intereses ajenos sin mandato de su dueño.

Sistema: Conjunto de cosas que relacionadas entre sí ordenadamente contribuyen a determinado objeto.

Información: Comunicación o adquisición de conocimientos que permiten ampliar o precisar los que se poseen sobre una materia determinada. Conocimientos así comunicados o adquiridos. Acción y efecto de informar.

Tratamiento de información: Aplicación sistemática de uno o varios programas sobre un conjunto de datos para utilizar la información que contienen.

Tratamiento de textos: Proceso de composición y manipulación de textos en una computadora.

Fuente de información: Confidencias, declaraciones o documentos que sirven de base para la elaboración de una noticia o reportaje periodístico. Personas que emiten esas declaraciones.

\section{Análisis y discusión}

\section{Gestión de información}

La información brinda poderío y posicionamiento a quien la utiliza actualmente. Es la materia prima para avanzar a un futuro conocido o al menos con una predicción de él, por esta razón debe gestionarse de manera eficiente, es decir seleccionarla, adquirirla, procesarla, validarla y entre otros pasos socializarla.

Para que la información pueda ser trasmitida dentro de la institución en el momento justo cuando cada área lo requiere y de manera directa a la persona correcta, deben utilizarse diferentes herramientas que faciliten el trabajo como son las antes mencionadas: gestión de información, vigilancia tecnológica, la inteligencia empresarial y la gestión del cocimiento entre otras. 
La integración de todas las áreas y sus funciones dentro de una institución se facilita mediante la flexibilidad, la rapidez y el flujo eficiente de la información en el sistema. Esto se logra con la integración de las nuevas tecnologías de la información y el conocimiento; por eso la gestión de información es el centro de este servicio, para trabajar de manera eficaz con los datos que se generan en cada uno de los sistemas de la entidad que se analiza.

La Gestión de Sistemas de Información conlleva un análisis exhaustivo de cada fuente de información, de tratamiento de textos, etc. que existe dentro del sistema, estos se evalúan, comparan, miden, con el objetivo de ofrecer soluciones a los problemas que puedan existir en ellos. Este tipo de gestión identifica las amenazas, anula las debilidades, aprovecha las oportunidades e incrementa las fortalezas, permite controlar los procesos que se ejecutan en la organización, ya sean de pago, producción, servicios, contabilidad, transporte, logística, marketing entre otros.

La gestión de sistemas de información aplicada por el CIGET a organizaciones del territorio permite la auditoría, control y evaluación de los recursos conque esta cuenta, y facilita los procesos y operaciones que las mismas desarrollan.

Por todo lo antes expuesto las autoras analizan la Gestión de información dentro de la Gestión de sistemas de información, donde esta es la columna vertebral, para que todo sistema funcione deberá mantener actualizada, organizada, controlada, verificada etc. toda la información que maneja.

Como todo servicio de consultoría de gestión de sistemas de información se ejecuta con ética, los profesionales que la llevan a cabo son conocidos como consultores, los mismos realizan un informe al finalizar la misma, que recoge los resultados obtenidos durante la evaluación de los sistemas estudiados. La información que se recupera es tratada de manera confidencial, y fidedigna, el trabajo del un consultor puede resultar complicado porque los integrantes de la organización cliente e ocasiones ocultan información, debido a que ven al consultor como alguien externo que se inmiscuye en su trabajo. En realidad el consultor sugiere soluciones a los problemas identificados, influye en la toma de decisiones por el directivo pero no participa directamente en los procesos que en un final se realizan en la organización para mejorar el funcionamiento.

El consultor evalúa, diagnostica, compara, sugiere, capacita pero no ejecuta el trabajo de los clientes.

Una vez concluido el servicio de consultoría deberán divulgarse los resultados ante los directivos de la entidad y si así se decidiera ante los trabajadores para la generalización de los estudios que se aplicaron con anterioridad y durante el transcurso de las tres etapas.

Características del servicio de consultoría:

- Objetivo

Realizar auditoría de los recursos de información que contiene cada sistema de la organización, identificar las fortalezas, debilidades, amenazas y oportunidades de cada sistema dentro de la empresa que se estudia.

- Alcance

Puede ser aplicado en empresas, organizaciones académicas o entidades de cualquier tipo.

- Requerimientos

Personas: Elemento fundamental para desarrollar las acciones que conlleva la gestión de sistemas, los diferentes procesos los ejecutan las personas, de su capacidad depende el éxito de cada acción que se emprende.

Tecnología: Para mejorar procesos y procedimientos, proporciona nuevas vías para compartir, almacenar, obtener y evaluar entre otros la información.

Información: Constituye la materia prima por excelencia, para obtener los resultados.

- Ventajas 
Permite la obtención de uno o más productos de información, un informe final del servicio de la consultoría, manual de sistemas de información, la evaluación de los recursos del sistema, se identifican los diferentes subsistemas que interactúan dentro de la organización cliente, además de la auditoría de información. Posibilita además la identificación de los recursos y su evaluación para su eficiente utilización.

\section{- Etapas}

Se desarrolla en tres etapas (diagnóstico, asesoría/diseño, implementación)

\section{Descripción de las etapas del servicio}

Un consultor como los que aplican el servicio que se describe, tiene como cliente a una organización, la estudia, analiza e identifica sus problemas y propone posibles soluciones. EI CIGET, parte de la siguiente estrategia:

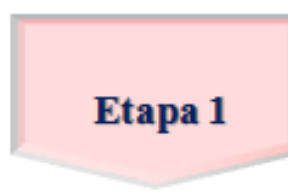

\section{Diagnóstico}

\section{Diagnóstico}

\section{Asesoría}

\section{Etapa 3}

\section{Etapa 2}

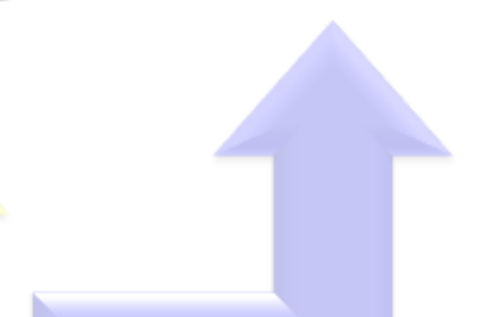


(Asesoría/ Diseño de servicios/productos) Según el resultado de los análisis, se investiga el servicio/producto que realmente pueda resolver las necesidades de información; en caso que el cliente no está claro de lo que realmente necesita.

Según lo encontrado y analizado previamente, se diseña un servicio que cumpla con los requisitos del correcto flujo organizacional, por tal razón siempre será necesario la elaboración del diagrama de flujo informacional.

Pronosticar un producto que responda a las necesidades, siendo este: una intranet corporativa, un sitio Web, una multimedia, un sistema de archivo, un centro documental etc.

- Estudio y análisis de los informes elaborados en la etapa anterior.

- Búsqueda y reconocimiento del servicio/producto que se va a desarrollar según corresponda con las deficiencias e ineficiencias encontradas, en bibliografía, ya sea en impresa o digital.

- Diseño del servicio/producto escogido para la entidad según necesidades estudiadas y encontradas.

(Implementación) Puesta en función del producto diseñado. Es la etapa de prueba piloto, correspondiente a la puesta en marcha del servicio, velar por su correcto funcionamiento y limar errores o deficiencias en caso que sean encontradas.

a) Explicar al personal el producto elaborado, en cuanto a:

- funcionamiento;

- uso;

- $\quad$ mantenimiento.

b) Evaluación del funcionamiento del producto entregado o prueba piloto.

Una vez administrados estos períodos y terminado el servicio se le entrega al cliente el aval de conformidad para que lo firme, donde valora la efectividad del servicio y explica si el mismo les proporcionó un desarrollo de las capacidades y mejoras en sus sistemas de información y en el personal de la empresa.

Algunos servicios y/o productos en un servicio de Consultoría Estratégica en la Gestión de Sistemas de Información

- Intranet Corporativa

- Multimedias

- Sistema de Archivo

- Cuadro de Clasificación Documental

- Sistemas de Gestión Documental

- Manuales de Información.

- Diagramas y Profesiogramas

- Matriz DAFO

- Plan de Acciones

- Listas de Contenidos 
- Compendios Informativos

- Lista de Resúmenes

- Servicios Tradicionales de una biblioteca (procesamiento analítico-sintético)

- Boletines Informativos

$\underline{\text { Importancia }}$

Radica en que el servicio de consultoría en Gestión de Sistemas de Información que se presenta brinda al cliente un análisis de las debilidades, amenazas, oportunidades y fortalezas de la organización, les brinda un diagnóstico e identifica las dificultades que afrontan y brinda posibles soluciones para el mejoramiento de la entidad.

$\underline{\text { Resultados }}$

$\stackrel{\leftrightarrow}{\rightarrow}$ Universidad de Ciencias Médicas de Guantánamo.

- Manual de Sistemas de Información

- $\quad$ Diagrama de Flujo de Datos con su correspondiente diccionario

- Lista de Recomendaciones para mejorar el funcionamiento de cada uno de los sistemas estudiados

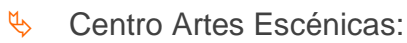

- Centro de Gestión Documental

- Boletín Informativo

- Lista de referencias

(4) Materiales de la Construcción

- Cuadro de Clasificación

- Archivo Pasivo o Histórico

Su aporte económico se fija teniendo en cuenta las etapas que deban aplicarse y la complejidad del servicio, puede variar el precio final según se concerta entre las partes y acorde al tipo de entidad cliente.

En cuanto a su aporte a la comunidad:

$\Leftrightarrow$ La consultoría que se describe en este trabajo permite responder a uno de los objetivos de trabajo del CIGET, el cual está relacionado con el diseño y perfeccionamiento de productos y servicios, encaminado a satisfacer las necesidades de los clientes.

$\stackrel{4}{4}$ Eleva la productividad de la actividad que realiza el cliente en cuestión porque lo ayuda a: identificar sus dificultades, aprovechar las oportunidades, eliminar las debilidades y enfrentar con éxito las amenazas.

$\stackrel{4}{4}$ En el ámbito científico aporta elementos a utilizar en el futuro para aplicarlo dentro del perfeccionamiento empresarial, como parte del sistema informativo con que deben contar las empresas insertadas en este proceso, debido a los puntos de contacto que tiene con el mismo.

\section{Conclusiones}

El servicio de consultoría permite responder a uno de los objetivos de trabajo del CIGET, con respecto al diseño y perfeccionamiento de productos y servicios, encaminado a satisfacer las necesidades de los clientes. 
Referente a las necesidades de información, se muestran ejemplos de servicios/productos que pueden ser creados 0 asesorados, según lo plantee la situación.

Se muestran sus beneficios, importancia y los resultados que se obtienen de su aplicación y sus características. En el ámbito científico, aporta elementos a utilizar en el futuro para aplicarlo dentro del perfeccionamiento empresarial, como parte del sistema informativo con que deben contar las empresas insertadas en este proceso, debido a los puntos de contacto que tiene con el mismo.

Eleva la productividad de la actividad que realiza el cliente, porque lo ayuda a identificar sus dificultades, al aprovechamiento de las oportunidades y eliminación de las debilidades y a enfrentar con éxito las amenazas.

\section{Bibliografía}

Dans, E. (2006). El departamento de sistema de información y su función corporativa: retos de futuro en investigación [Versión Electrónica]. Recuperado de: http://profesores.ie.edu/enrique dans/download/retosdefuturo.pdf

Fernández, J. B. R. (2003). La gerencia de información: El caso de los archivos. [Versión Electrónica]. BIBLIOS, 3-13 Recuperado de: http://dialnet.uniroja.es

Los sistemas de información y su importancia para las organizaciones y empresas. (2001). [Versión Electrónica]. Recuperado de: http://www. monografias.com/trabajos24/tics-empresas/tics-empresas.shtml

Mendoza Barrón, María Caridad. (2006). La importancia de la función de consultoría. 3-13 [Versión Electrónica] VERITAS. Recuperado de: http://www.ccpm.org.mx/veritas/abril2010/importancia consultoria.pdf

Ministerio de Justicia. (2007). Gaceta Oficial No. 041 Extraordinaria de 17 de agosto de 2007. La Habana. Recuperado de: http://www.gacetaoficial.cu/html/pdf/GO X 041 2007.pdf

Ponjuán, G. e. a. (2004). Sistemas de información: Principios y aplicaciones. La Habana.

Sistema de información. (2002). [Versión Electrónica]. WIKIPEDIA. Recuperado de: http://es.wikipedia.org/wiki/Sistema de informaci\%C3\%B3n 


\section{ANEXO}

\section{Servicio de Consultoría Estratégica}

Descripción: Se basa en la realización de estudios que permiten al cliente la identificación y solución de problemas y la toma de decisiones estratégicas en cualquier sector, basados en la obtención de la información. Para la gestión del conocimiento.

Objetivo: Identificación, obtención, tratamiento y análisis de la información y el conocimiento para la toma de decisiones estratégicas.

\section{Las consultorías estratégicas que brindamos son:}

\subsubsection{Inteligencia Empresarial}

34.06.18 Gestión de Sistemas de Información

34.06.19 Diseño de Plan de Manejo

\section{Destinatarios de este servicio:}

Organizaciones de cualquier sector económico, industrial o académico con independencia del producto o servicio que oferte, que estén interesadas en mejorar su desempeño.

\section{Beneficios que obtendrá la empresa:}

- Entidad mejores preparadas para la toma de decisiones empresariales.

- Desarrollo de capacidades en inteligencia empresarial en el personal de la empresa.

- Sistemas de trabajo mejor organizados y con resultados óptimos.

- Mejora en la toma de decisiones.

- Inventario y clasificación de las existencias de desechos peligrosos

\section{Etapas en que se desarrolla el servicio:}

Etapa No.1: Diagnóstico

En esta etapa se realiza un análisis interno de la información así como de la documentación, además de la evaluación del comportamiento humano con respecto al desarrollo de los sistemas como medio de entrada y salida de información y/o servicios y productos y de los desechos que maneja la institución.

\section{Esta etapa tiene una duración de 30 días.}

\section{Etapa No. 2. Diseño}

Según la evaluación realizada en la etapa anterior se establecerá un plan de acción. Se desarrollan productos como diagramas de flujos, manuales de información, perfiles estratégicos, diccionarios de datos, plan de manejo, entre otros.

\section{Esta etapa tiene una duración de 30 días.}


Etapa No.3. Implementación

Aplicación del diagrama de flujo, manuales de información, evaluación del funcionamiento del sistema

Tiempo de duración del servicio. 30

El servicio completo podrá realizarse en un período de 90 y un valor de $X$

\section{Dados da autora}

\section{Zunilka Limonta Favier}

Licenciada en Bibliotecología y Ciencia de la Información, graduada en la Universidad de Camagüey, Cuba. Profesor Instructor. Especialista de Gestión Documental y Webmaster en la Universidad de Guantánamo, Cuba.

zunilka@ciget.gtmo.inf.cu

\section{Evelyn Milagro Otero Caballero}

Licenciada en Bibliotecología y Ciencia de la Información, graduada en el Instituto Superior Minero Metalúrgico de Moa (ISMM). Especialista en análisis, procesamiento y servicios de información en Centro de Información y Gestión Tecnológica (CIGET), Guantánamo, Cuba.

evelyn@ciget.gtmo.inf.cu

\section{Daymara Mediaceja Corona}

Licenciada en Bibliotecología y Ciencia de la Información. Especialista en análisis, procesamiento y servicios de información en Centro de Información y Gestión Tecnológica (CIGET), Guantánamo, Cuba.

daymara@ciget.gtmo.inf.cu

\section{Briseys Calvo Coello}

Licenciada en Bibliotecología y Ciencia de la Información, graduada en la Universidad de Camagüey, Cuba.

briseyscc@cug.co.cu

Recebido-Received : 2012-11-30

Aceito - Accepted : :2013-06-30

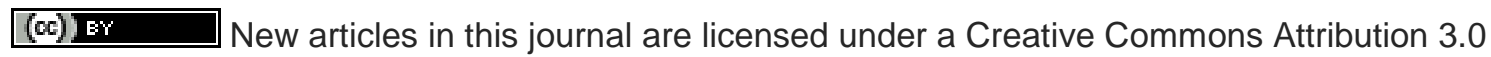
United States License.

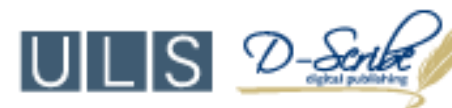

This journal is published by the University Library System of the University of Pittsburgh as part of its D-Scribe Digital Publishing Program and is cosponsored by the University of Pittsburgh Press. 